\title{
Les âges de George Sand
}

\author{
Fredrik Westerlund (Dép. des Langues étrangères et traductologie - \\ Université de l'Est de la Finlande)
}

\begin{abstract}
The article discusses ageing and old age in three of George Sand's texts: Indiana (1832), La Mare au diable (1846) and the first part of the novel Consuelo (1842). I use the first two parts of Pat Thane's subdivision of age into a corporal, a cultural and a chronological component.

In Sand's fiction, the ageing female body withers, while the male body is worn. There are various reasons behind the decline. If the characters age of worries and trouble, the process can be reversed, and the persons can regain youth - at least partly - when the troubles go away.

In a cultural perspective, the living conditions vary substantially between classes, specifically if the characters need to work for their living or not. Among peasants and workers, the tolerance for the age gap between spouses is narrower than in the bourgeoisie. The former risk to encounter poverty and need if the husband grows old sooner than the wife, while an elderly man of the bourgeoisie can marry a young woman in order to preserve her social status.

In both classes, characters considered as old, while wise and experienced, do not longer interest anyone. Death is their future, and they ridicule themselves if they initiate long-term projects. Another stereotype, the old fool, appears as well, but in the case of Madame Carjaval, it is a role she plays to protect her niece.

Many of the attitudes towards old people still exist today. The main difference vis-à-vis George Sand's time is that, due to the development of longevity, old age arrives to people later now than in the $19^{\text {th }}$ century.
\end{abstract}

Keywords: Old age, George Sand, corporal age, cultural age, Indiana

\section{Introduction}

Dans cet article, nous nous proposons d'explorer, dans une perspective thématique et symbolique, le thème de la vieillesse, du vieillissement, et accessoirement de la jeunesse dans l'œuvre de fiction de George Sand, à partir d'un corpus constitué de trois textes, les romans Indiana (1832) et La Mare au diable (1846), ainsi que le premier tome du long roman Consuelo (1842). Les personnages principaux de ces trois récits sont des jeunes femmes mariées ou en âge de l'être, c'est-à-dire entre seize et dix-huit ans au début de l'histoire.

Nous portons un intérêt particulier aux évocations des caractéristiques, qu'elles soient thématisées ou, plus encore présupposées comme des évidences. Sous cette deuxième catégorie tombent les associations d'apparence gratuite - le cas typique est l'attribution de la beauté à la jeunesse, très fréquente dans les textes du corpus. 
Selon Pat Thane (2000) la notion de vieillesse se subdivise en trois composants : Le premier, fonctionnel concerne la capacité et l'aptitude à accomplir des tâches différentes, l'autre culturel, traite de la manière dont les êtres humains se comportent ou sont considérés à partir d'une définition subjective en tant que vieux et en troisième lieu le composant chronologique, qui correspond à l'écoulement absolu du temps - c'est " le décompte des années ». C'est avant tout l'aspect culturel que la littérature donne à lire, surtout chez une auteure comme George Sand, très sensible aux convenances et aux rôles attribués aux femmes, hommes et enfants dans la société de son temps. Il faut pourtant mettre en garde contre une conception trop monolithique de la notion de société, d'abord à cause de son évolution rapide à l'époque de Sand, avec l'industrialisation, l'urbanisation et la démocratisation, et en raison des différences dans les conditions de vie des membres de différentes couches sociales: Indiana est bourgeoise, Germain et Marie sont paysans et Consuelo se situe à cheval entre le prolétariat et la bourgeoisie, voire l'aristocratie : tout en étant censée gagner son propre pain, elle intègre les cercles plus élevés de la société. Les actions des romans se déroulent par ailleurs dans des espaces très différents : la vie parisienne d'Indiana contraste avec celle qu'elle a connue sur l'île Bourbon, Germain et Marie habitent la campagne française, tandis que Consuelo fait ses débuts à Venise, puis voyage en différents endroits.

Si l'on regarde au-delà des disparités et des cas particuliers, les personnages de George Sand semblent rentrer dans la vieillesse à partir de quarante ans pour y arriver définitivement avant soixante ans. On fait pourtant l'esquisse d'un avenir possible jusqu'à quatre-vingts ans, mais c'est alors un très grand âge, surtout au XIX ${ }^{\mathrm{e}}$ siècle où l'âge moyen de la mortalité tournait autour de 50 ans. D'autre part, les personnages peuvent conserver des traits enfantins durant leur âge adulte entier. Les soucis, le travail ou la débauche aidant, les personnages peuvent démontrer des signes de vieillesse - peau mate, apparition flasque et usée, cheveux gris peu après leurs vingt ans.

Nous traiterons de la représentation des âges - et notamment de la vieillesse - chez George Sand en deux étapes, suivant le schème de Thane qui distingue la vieillesse comme état corporel et comme phénomène culturel. D'abord, notre attention se porte sur la vieillesse comme état corporel.

\section{La vieillesse comme état corporel}

La vieillesse est habituellement associée aux risques de maladies et d'infirmités, à une réduction de la capacité physique des personnages. Le chercheur suédois Lars Tornstam $(2011,108)$ a démontré qu'il s'agit de représentations stéréotypées généralement erronées. À l'époque contemporaine en effet, l'état corporel des personnes âgées et leur intégration sociale démentent ces clichés, ce qui s'explique par l'évolution des sociétés qui offrent de meilleures conditions de vie, la prise en charge des personnes âgées par la sécurité sociale, par exemple.

Globalement, George Sand se tient proche des stéréotypes. Dans le corpus, le vieillissement se fait voir dans l'évocation d'une transformation aussi bien physique que morale. Ainsi la vieillesse des personnages masculins se manifeste-t-elle par la couleur grise ou blanche de leurs cheveux ou par leur calvitie. Le vieux professeur Porpora dans Consuelo a des yeux nacrés, des pommettes tachetées de rouge (6). Il semble «crochu et maussade » (28), tandis que le comte Christian de Rudolstadt du même roman est pâle et morne (189) avec des joues flétries (196) des «membres amaigris » (298), une «santé affaiblie » (334). 
Consuelo le contemple qui prie : «Un vent frais [...] agitait autour de sa nuque une demicouronne de cheveux argentés ; et son vaste front, dépouillé jusqu'au sommet du crâne, avait le luisant jaunâtre des vieux marbres. » (298) Il porte non seulement les signes extérieurs de la vieillesse, mais son apparition évoque les caractéristiques d'un buste antique, ce qui souligne le caractère éternel du personnage. Le parallèle entre vieillesse et minéralité est à noter comme un trait caractéristique dans l'œuvre de Sand. Les informations sur l'âge dénominatif du vieux comte sont contradictoires : d'une part, le texte affirme que sa sœur ainée est sexagénaire (189), et d'autre part que son fils qui fête ses trente ans est le sixième enfant de ses secondes noces, conclues à l'âge de quarante ans. (217-218) Nous voyons dans cette confusion que l'idée de la vieillesse l'emporte sur l'exactitude de l'âge chronologique.

Dans le corpus, les deux caractéristiques associées à la vieillesse sont notamment l'usure et le flétrissement. Celui qui est usé est vieux, celle qui est flétrie n'est plus jeune.

Au début du roman Indiana, le colonel Delmare, le vieux mari de la protagoniste, est présenté comme un «homme jadis beau, maintenant épais, au front chauve, à la moustache grise, à l'œil terrible » (6-7). Il est «vieux et cassé » (400). En plus, il a de l'humeur, le temps étant humide, il souffre de rhumatismes. Bref, «son front chauve et son corps affaibli annonçaient une existence chancelante, une vie usée » (290). La figure du colonel accumule les stéréotypes de la vieillesse: santé douteuse, humeur, calvitie, nostalgie d'une beauté perdue.

La Mare au diable est, des trois textes, celui où la vieillesse et les âges sont le plus thématisés. Selon Germain, un paysan de vingt-huit ans, protagoniste masculin « [...] les années ne font pas toujours l'âge. Cela dépend de la force et de la santé qu'on a. Quand un homme est usé par trop de travail et de misère ou par la mauvaise conduite, il est vieux avant vingt-cinq ans. » (107). Tandis que Germain est encore à l'âge de se remarier, ce qui équivaut à être relativement jeune, il admet qu'il existe d'autres hommes qui, tout en étant ses cadets, sont «vieux ». Dans les remarques concernant la vieillesse corporelle des hommes de vingt ans s'entrevoit la critique sociale de George Sand, préoccupée par les conditions de vie des petits gens. Germain appartient à la catégorie des paysans pauvres contraints de labourer la terre pour survivre et pour qui chaque bouche supplémentaire à nourrir constitue un fardeau considérable. On est loin de la bourgeoisie urbaine des deux autres romans dont les membres vivent de rentes et d'investissements.

Dans Consuelo, l'adjectif qualificatif «vieux» est toujours associé au ténor quadragénaire Stefanini $(141,156)$. Son tort a été «d'attendre pour prendre sa retraite que l'âge eût exténué sa voix et enlaidi son visage ». L'antéposition de l'épithète «vieux » souligne que l'évocation de la vieillesse du ténor est l'évidence. Physiologiquement parlant, l'exténuation de la voix est le résultat de l'usure plutôt que de l'âge en soi, tandis que l'association de la laideur à la vieillesse à quarante ans reflète les attentes du public qui demande incessamment des artistes toujours plus jeunes. Néanmoins, il s'agit de modifications physiologiques venant avec l'âge et peut-être avec «la mauvaise conduite » évoquée par Germain. Sand décrit en effet la vie d'artiste comme une vie de désordre, remplie de veillées, libations et intrigues.

Si les signes de la vieillesse masculine se lisent principalement sur le visage et dans les cheveux, la vieillesse féminine s'inscrit plus largement dans le corps, et avant tout dans la 
peau, qui est sèche. La chanoinesse Wenceslawa de Rudolstadt que rencontre Consuelo est un «respectable personnage sexagénaire affligé d'une bosse énorme et d'une maigreur effrayante » (189), dont la longue main est «sèche et luisante comme de l'ivoire jaune » (287). Tout comme la couleur du chef de son frère est associée au marbre, pour la main de Wenceslawa, le texte fait un autre rapprochement minéral, celui de l'ivoire. Les références parsemées au minéral pourraient être un vestige de l'intérêt documenté de George Sand pour les minéraux et pour la géologie. Dans le contexte de la vieillesse, les parallèles évoquent ce qui est éternel et durable. Dans Indiana, la mère du coureur de femmes Raymon lui tend également une «main sèche et diaphane » (327), tandis que, dans le roman éponyme, celle de Consuelo, épuisée de fatigue (56), est réduite à l'air d'un spectre (62). En allant au bal, la tante d'Indiana, Madame de Carvajal est «roide, parée comme un portrait de Van Dyck» (84).

Ce sont plutôt les soucis et le malheur, avant tout celui de l'amour, qui font vieillir les femmes. Indiana, qui a à peine vingt ans, associe explicitement la souffrance au vieillissement en disant à Raymon : «vois comme je suis pâle : comme j'ai vieilli, comme j'ai souffert. » (457). La pâleur, conséquence de la souffrance morale est donc un signe de vieillissement, mais, heureusement, ce processus est réversible. Quelques années après cette scène, un jeune voyageur rencontre une Indiana «fraîche » «si belle, si jeune (car elle semblait avoir à peine dix-huit ans) » (528). Et le voyageur d'expliquer : «l'image d'un cœur brisé par les chagrins : quand le bonheur vient le retrouver, il s'épanouit et se rajeunit bien vite. » (532) De cette manière, le vieillissement corporel déclenché par la souffrance morale est transmué en un positionnement social et affectif où la souffrance vieillit et où le bonheur rajeunit.

Parfois, jeunesse et vieillesse peuvent cohabiter dans le même corps. Ainsi Ralph, cousin d'Indiana et son fidèle serviteur platonique est-il décrit comme « un homme dans toute la force et dans toute la fleur de la jeunesse, et dont les joues brillantes, la riche chevelure d'un blond vif, les favoris bien fournis, juraient avec les cheveux grisonnants, le teint flétri et la rude physionomie de patron » (9). Indiana affirme qu'il est vieux à vingt-neuf ans : «son visage est jeune, mais son cœur est usé à force d'avoir souffert » (210). Dans le premier passage, la caractéristique masculine de vieillesse par excellence, les cheveux grisonnants, est combinée au flétrissement de la peau. Chez Ralph, la souffrance a provoqué une usure du cœur qui l'a vieilli.

Pour la mère de Consuelo, pauvre et vagabonde, son corps porte encore en vieillissant des vestiges d'une beauté passée. "Cette femme doit avoir été belle » (65) dit-on dans les cafés où elle chante. Et Consuelo de conclure : «la beauté est comme cela quand on est pauvre; c'est un instant : on n'est pas belle encore, et puis bientôt on ne l'est plus. » (64-65) Dans l'affirmation de Consuelo, où statut économique et beauté sont mis en relation, l'engagement social de l'écrivaine s'entrevoit d'une manière exceptionnellement explicite.

Dans le corpus, derrière la dégradation du corps, il y a l'usure. Celle-ci, à son tour, résulte de deux facteurs : l'écoulement irréversible du temps ainsi que la souffrance, physique ou psychique. Souvent, les deux vont de pair, les personnes âgées ayant beaucoup de soucis, avant tout pour leur postérité et pour leur santé. Le vieillissement résultant du deuxième facteur est réversible - une fois les soucis passés, le personnage peut rajeunir. 
Après cette association qui met à nu la distinction des deux processus séparés qui font physiquement vieillir les personnages, nous passons à la deuxième forme de vieillesse selon la classification tripartite de Thane, à savoir la vieillesse comme phénomène culturel.

\section{La vieillesse comme phénomène culturel}

Le phénomène culturel de la vieillesse comprend d'abord le comportement et les attitudes des vieilles gens. Les qualificatifs employés pour les décrire ne sont pas toujours très flatteurs. Le mari d'Indiana, le vieux Delmare a de l'humeur. Il est jaloux, irascible et violent. De toute apparence, c'est par des raisons pratiques qu'il a épousé Indiana, qui est sa cadette de plus de quarante ans :

[I]l se sentait vieux, les soins de sa femme lui devenaient chaque jour plus nécessaires. Il se faisait une terrible peur de la solitude, et [...] la réflexion le ramenait bientôt à cette faiblesse des vieillards qui s'épouvante de l'abandon. Trop affaibli par l'âge et les fatigues pour aspirer à devenir père de famille, il était resté vieux garçon dans son ménage, et avait pris une femme comme il eût pris une gouvernante. [...] et s'il s'affligeait de ne pas régner sur ses affections, c'était parce qu'il craignait d'être moins bien soigné sur ses vieux jours. (412-413)

Dire que, dans cet exemple, Delmare se sent vieux constitue un exemple d'une vieillesse psychologique. Dans le cadre de cet article, nous n'avons pas trouvé nécessaire de séparer le vieillissement psychologique du vieillissement culturel : si quelqu'un se sent vieux, c'est plutôt par rapport aux habitudes de la société qui l'entoure ou bien, comme c'est le cas ici, en contraste avec la jeunesse d'un proche.

À un moment de repentir, l'insouciant Raymon décide de se consacrer à «sa mère âgée, débile $[\ldots]$ cette pauvre femme $[\ldots]$ qui ne vivait plus que pour lui, son unique bien, son seul espoir » (151). Le fils est donc censé soigner la mère, faute de bru ou de fille.

Le mariage entre Delmare et Indiana est possible grâce à leur statut social de bourgeois relativement aisés. Colonel à la retraite, industriel, le mari âgé d'Indiana n'a pas besoin de travailler pour subvenir à ses besoins. La situation est toute autre dans La Mare au diable où les paysans Marie et Germain tombent amoureux l'un de l'autre. En essayant de la convaincre du raisonnable dans leur alliance il l'exhorte à oublier son âge, puis «Pense que c'est une fausse idée qu'on se fait quand on croit qu'un homme de trente ans est vieux. D'ailleurs je n'ai que vingt-huit ans ! (107). Marie, qui en a seize se souvient des paroles de sa mère :

$[\mathrm{U}]$ ne femme de soixante ans est bien à plaindre quand son mari en a soixante-dix ou soixante-quinze, et qu'il ne peut plus travailler pour la nourrir. Il devient infirme, et il faut qu'elle le soigne à l'âge où elle commencerait elle-même à avoir grand besoin de ménagement et de repos. C'est ainsi qu'on arrive à finir sur la paille. (108)

La jeune paysanne semble bien sage de baser son argument sur des événements potentiels à quarante ou cinquante années de distance. Alors qu'une différence d'âge de dix ou quinze ans est difficile à gérer chez les paysans qui vivent du travail de leurs mains, la différence d'âge quadruple entre Indiana et son mari s'accorde parfaitement avec les us et coutumes de la société. Le colonel a bien le droit, et il a eu la possibilité d'épouser la jeune fille, à qui il s'adresse « sur un ton moitié père, moitié mari » (15). On comprend que la raison 
principale de cette union est le souci de préserver à Indiana son statut social de fille bourgeoise, qui n'est pas censée travailler pour vivre. Le côté affectif ne rentre pas dans le calcul, et Indiana, mariée à seize ans au sexagénaire Delmare, attend la mort de son mari afin de pouvoir épouser son amour Raymon, son aîné de sept ans.

Les personnes âgées des trois romans sont très conscientes du fait qu'elles n'intéressent plus personne. Dans Indiana, Sand parle de «la secrète satisfaction de la vieillesse qui se venge en réprimant les plaisirs des autres »(77), et Madame Carvajal constate qu'on ne la recherche que pour approcher sa nièce (84). De cette manière, le vieillissement social à l'époque de George Sand comporte une fatale diminution d'attractivité. Cette idée, pourtant atténuée, persiste encore de nos jours.

L'avenir appartient aux jeunes gens, et non pas aux vieillards. Ceux qui, à l'instar du vieux Delmare, se font des projets pour l'avenir, tombent dans le ridicule. Raymon sourit intérieurement « de l'entendre parler de dix ans comme d'un jour lorsque son front chauve et son corps affaibli annonçaient une existence chancelante, une vie usée. » (290). On ne peut pourtant compter sur la mort imminente du vieillard, et ce même Raymon ne va pas attendre qu'Indiana soit délivrée de son vieux mari, car celui-ci pourra vivre vingt ans encore et donc atteindre, voire dépasser quatre-vingts ans.

Parallèlement, ce qu'on déplore dans le suicide à dix-neuf ans de Noun, sœur de lait d'Indiana, c'est sa beauté, sa vivacité, mais avant tout le fait qu'elle aurait eu toute sa vie devant elle. Autrement dit, la mort appartient aux vieillards et pas aux jeunes gens.

Traditionnellement, la vieillesse est associée à la sagesse et au savoir, souvent acquise par une longue expérience. Ceci est le cas du maître Porpora dans Consuelo, qui enseigne la musique aux jeunes filles depuis plus de quarante ans, ce qui laisse entendre que, en connaissant celles qu'il a déjà éduquées, il en sait long sur le comportement de ses élèves présentes et futures.

$\mathrm{Au}$ bal où se rend Indiana figure « une femme qui connaissait tout le monde, et qui remplissait dans les réunions le rôle d'almanach » (64). Elle puise son savoir de sa longue expérience de la vie mondaine. Enfin, Consuelo reçoit les paroles du comte Christian, « ce saint vieillard» comme «un baume céleste»(291-292), ce qui ouvre la pensée à la représentation des vieilles gens comme des personnes qui, par leur proximité avec la mort, possèdent déjà de leur vivant quelques connaissances réservées autrement aux trépassés. Son fils, le comte Albert est jugé vieux en entrant dans sa trentième année, non moins à cause de ses propos que son entourage prend pour de la folie alors qu'ils traduisent des visions d'un audelà. C'est ainsi que ce sage qui, en raison de son âge trop peu avancé, revêt un rôle social qui ne lui revient pas encore, est perçu comme un fou.

La limite entre sagesse et folie est donc poreuse - si les personnes âgées disent des vérités non attendues, ou s'ils nourrissent des projets qui n'intéressent pas leur entourage, on les écarte comme de vieux fous. C'est le sort de Madame Carvajal, qui fait des réponses ambiguës, voire incongrues, pour protéger sa jeune nièce d'un scandale. Elle sacrifie une partie de son statut social en assumant le rôle de vieille folle. Les propos de maître Porpora, entraîné dans les intrigues amoureuses et ambitieuses de la Corilla, sont qualifiés de «lubies de ce vieux fou » (131) par elle, même si c'est lui qui fait entendre raison dans la situation. 
Pour l'instance narrative l'expérience liée à l'âge est une source de connaissance. Dans une de ses rares adresses directes au lectorat, l'auteur dit : «— Tu me diras, cher lecteur, que tu n'as guère connu de ces organisations exceptionnelles. Je te répondrai, lecteur bien-aimé, que je n'en ai connu qu'une seule, et si, suis-je plus vieux que toi. » (57)

Ici, la narration puise donc son autorité dans l'expérience qui est venue à l'auteur avec l’âge, supérieur à celui du lecteur.

Dans cet article, nous avons étudié la vieillesse et le vieillissement dans les textes de George Sand sous trois perspectives différentes suivant Pat Thane.

Dans le corpus, les descriptions de l'âge physique sont assez conventionnelles. Le vieillissement est une usure du corps, provoquée par le travail, la débauche ou les chagrins. Cette usure ne coïncide qu'en partie avec l'écoulement des années. Vieillir, c'est aussi flétrir, et ce processus, souligné par des parallèles avec le monde végétal, est plus étroitement lié au cours du temps. Notons pourtant l'emploi des parallèles entre vieillesse et minéralité, qui est une particularité de George Sand. Chez Sand, le vieillissement est beaucoup plus naturel et moins traumatique que chez par exemple André Gide, pour qui le vieillissement représente un renoncement et un déclin inévitables qu'il faut s'efforcer d'accepter.

Pour ce qui est de l'âge social, les personnages de George Sand semblent bien cantonnés dans les rôles respectifs qui leur sont imposés par l'âge, le sexe et la classe sociale. Rien d'étonnant en soi - le contexte historique va du milieu du dix-huitième siècle dans Consuelo jusqu'aux premières décennies du dix-neuvième siècle dans Indiana. On entrevoit quand même dans des mots anodins comme «encore » et « déjà » que certains personnages ont tendance à sortir du rôle dans lequel ils sont confinés par leur âge.

Dans le corpus, l'enfance et la vieillesse se chevauchent et se dépassent. Tout en étant relativement éparses, les chronologies directes donnent quelque peu d'information. Nous avons déjà cité les propos de Germain, où il explique qu'un homme peut être vieux à vingt-cinq ans, s'il est usé par le travail ou par la débauche. Il arrive aussi que la narration évoque des personnages qui ont dépassé le cap de vingt ans depuis quelque temps comme des « enfants ». D'autre part, les personnes d'un certain âge peuvent très bien raisonner ou se comporter comme des enfants. Ainsi dans Indiana Delmare démontre-t-il une « obstination aveugle d'un enfant de soixante ans » (74). Le vieillissement est en relation au climat aussi. Dans le même roman, la narration fait la comparaison de l'île Bourbon d'où proviennent Indiana et Noun à la France, pour conclure que « dix-neuf ans de l'île Bourbon [...] équivalent à vingt-cinq ans de notre pays » (70), et à dix ans la créole Noun n'est plus une enfant mais déjà une jeune fille (501).

À l'instar de nos attentes, nous avons constaté que la vieillesse survient plus tôt dans les textes de George Sand que dans des textes plus tardifs, tels par exemple ceux d'André Gide et de Jean-Marie Le Clézio, pour ne citer que deux exemples d'auteurs plus contemporains. Chez Sand, on est tout à fait vieux à soixante ans, et on ne vit plus au-delà de quatre-vingts ans. André Gide est moins précis quant au commencement de la vieillesse tout en déplorant qu'il semble exister des activités auxquelles il n'est pas censé s'abandonner à soixante-dix ans passés, telles les promenades en voiture ouverte. Dans la nouvelle «Un jour de vieillesse » de Le Clézio, qui raconte le dernier jour de Maria Vanoni, celle-ci est très vieille avant quatre-vingts ans. Rappelons que, encore dans les années soixante, Simone de Beauvoir emploie le terme «vieillard» pour désigner les personnes sexagénaires. À notre 
avis, la différence entre les trois auteurs concernant l'âge d'entrée dans la vieillesse chronologique s'explique principalement par des facteurs extérieurs: avec l'évolution technique, le progrès de la médecine et l'amélioration des conditions de vie, la longévité s'accroit toujours depuis le milieu du XIX ${ }^{\mathrm{e}}$ siècle.

Si l'on voulait élargir et approfondir cette recherche, il y a deux pistes qui s'imposent.

D'abord celle de la jeunesse. Vieillesse et jeunesse se chevauchent dans le texte de Sand, parfois jusqu'à la confusion, comme c'est le cas du flegmatique rêveur trentagénaire Albert de Rudolstadt, qui «mène dès à présent la vie tranquille et inoffensive des vieillards : ce sera le premier des Rudolstadt qui n'aura point eu de jeunesse. » (232)

L'autre piste à explorer est celle des relations familiales. L'absence des pères et des mères dans les romans étudiés est frappante. Le père de Consuelo est inconnu, tandis que sa mère, morte avant le commencement du récit, est seulement racontée, principalement par le relais des pensées et propos de sa fille. À l'âge de dix-huit ans, Anzoleto, l'ami de Consuelo, est orphelin. Dans la première partie du texte, seul le maître Porpora - pourtant sans enfants affiche des sentiments paternels. Arrivée au château de Schreckstein, Consuelo découvre une famille constituée de deux frères et une sœur ainsi que le fils du frère aîné, fiancé à la fille du frère cadet, une alliance de raison à laquelle celle-ci s'oppose de tout son cœur. Leurs mères sont mortes.

Indiana a été mariée au colonel Delmare après être devenue orpheline à seize ans. Son cousin dévoué Ralph est veuf depuis plusieurs années, et son enfant est mort avant le début du récit. Germain dans La Mare au diable est veuf aussi, et ce sera sa deuxième épouse, Marie, qui s'occupera de ses enfants. Dans le contexte de la vieillesse et des âges en général, ce manque de coexistence de générations successives et en conséquence l'absence de relations entre parents et enfants a des implications qui mériteraient bien d'être explorées.

\section{Bibliographie}

De Beauvoir, Simone, 1970. La Vieillesse. Paris : Gallimard.

Sand, Georges, 1832. Indiana. https://fr.wikisource.org/wiki/Indiana/Texte_entier

Sand, Georges, 1842. Consuelo. Tome I. https://fr.wikisource.org/wiki/Consuelo/Texte entier

Sand, Georges, 1999 (1846). La Mare au diable (1846), édition présentée, établie et annotée par Léon Cellier. Paris : Gallimard.

Thane, Pat, 2000. Old Age in English History. Oxford : Oxford university press.

Tornstam, Lars, 2011. Åldrandets socialpsykologi. 8., [rev. och utökade] uppl. Stockholm : Norstedts. 